\title{
Original
}

\section{Analysis of heat shock protein 70 and in vitro apoptosis in B-chronic lymphocytic leukemia cells by flow cytometry}

\author{
Tsunayuki Kakimoto, Atsuko Nagasawa, Taijiro Ishiyama \\ Kunihiko FuKuChI ${ }^{*}$ and Nobuyoshi TsuruokA
}

\begin{abstract}
To study the role of heat shock protein 70 (hsp70) in apoptosis of B-chronic lymphocytic leukemia (B-CLL), We in vestigated intracellular hsp 70 and bcl-2 protein expression and sensitivity of B-CLL cells to apoptosis in vitro in comparison with normal lymphocytes and acute lymphoblastic leukemia (ALL) cells by flow cytometry. We found that intracellular hsp 70 expression was significantly higher $(\mathrm{p}<0.05)$ in $\mathrm{B}-\mathrm{CLL}$ cells than in normal lymphocytes and ALL celIs. However, intracellular bcl-2 protein expression was not significantly higher $(p=0.0504)$ in $B-C L L$ cells than in normal lymphocytes. In contrast, the sensitivity of B-CLL cells to apoptosis was higher than that of normal B lymphocytes or ALL cells. These results show the discrepancy between high expression of hsp70 and bcl-2 protein and high sensitivity of B-CLL cells to apoptosis. It is important to consider the role of hsp70 and bcl-2 protein in the pathogenesis of apoptosis in B-CLL.
\end{abstract}

Key words : heat shock protein 70 , bcl-2, B-CLL, apoptosis, flow cytometry

\section{Introduction}

The family of heat shock proteins is highly conserved in evolution and can be induced in response to a variety of environmental stresses ${ }^{1)}$. In particular, heat shock protein 70 (hsp70) is constitutively expressed within cells and protects against environmental stresses ; and hsp70 is a molecular chaperone, anti apoptotic protein which along with bcl-2, plays an important role in programmed cell death (apoptosis) ${ }^{3,4)}$. In fact, hsp70 is overexpressed in some tumor cells ${ }^{5)}$. Expression of hsp 70 inhibits quercerin-induced apoptosis in leukemia cell lines ${ }^{6)}$. Furthermore, the susceptibility of acute myeloid leukemia (AML) cells to in vitro apoptosis correlates with hsp70 overexpression ${ }^{7)}$. To determine whether hsp70 plays an important role in apoptosis in B-chronic lymphocytic leukemia, we investigated the relations between intracellular hsp70 and bcl-2 protein expression and the sensitivity of lymphoid leukemia cells harvested from patients to apoptosis in vitro. we found that the high sensitivity of B-CLL cells to apoptosis in vitro correlated with hsp70 and bcl-2 protein overexpression. 


\section{Materials and methods}

\section{Patients}

Twelve patients with B-CLL (ten men, two women, aged 50-76 years) and seventeen patients with acute lymphoblastic leukemia (ALL) (five men, seven women, aged 17-80 years) were included in this study. The diagnosis of B-CLL was done according to the criteria of the International CLL Workshop ${ }^{8)}$. Six patients were diagnosed as stage A, two as $B$, one as $C$ and three are unkwoun. Only one patient had received no treatment; oth ers had received some treatment more than one year earlier. The percentage of leukemic cells in all samples was more than $75 \%$. ALL was diagnosed according to the FrenchAmerican-British (FAB) classification of acute leukemia ${ }^{9)}$. Eleven patients were at initial diagnosis and six were at relapse. Six patients were L1 and seven were L2. Samples from all patients were collected at presentation, before chemotherapy. The percentage of leukemic cells was more than $90 \%$.

\section{Leukemia cell isolation}

B-CLL cells from twelve patients were collected from peripheral blood. peripheral blood or bone marrow blood from seventeen cases of ALL was collected in heparin. Mononuclear cells were harvested from normal and leukemic blood on Ficoll Hypaque (Lymphoprep, Nycomed Pharma, Oslo, Norway) by density qradient centrifugation and were washed twice in RPMI 1640 by centrifugation at $2500 \mathrm{rpm}$ for 5 minutes. Cells were resuspended at 1 $\times 10^{6} / \mathrm{ml}$ in RPMI 1640 containing $10 \%$ fetal calf serum.

\section{Antibodies}

Monoclonal mouse anti-human bcl-2 oncoprotein, IgG1, kappa (Dako, Glostrup, Denmark) were generated by immunization with a synthetic peptide comprising amino acids 41 to 54 of human bcl-2 protein. Mouse serum (Dako) was used as an irrelevant control. Monoclonal mouse anti-heat shock protein 70 antibody (IgG1, Affinity Bioreagents, Golden Co) is located between amino acids 504 to 617 of human hsp70. Goat IgG was used to block a second antibody. Fluorescein isothiocyanate (FITC)-labeled goat antimouse IgG $(\mathbf{H}+\mathbf{L})$ was used as a second antibody for bcl-2 oncoprotein and hsp70 antibody staining. Propidium iodide (PI) was used for red fluorescence. Phycoerythrin (PE)-conjugated antibody for two-color immunofluorescence included Leu-19 (antiCD19, Becton Dickinson Immunocytometry Systems, San Jose, California) monoclonal antibody, and FITC-labeled human recombinant Annexin V (Caltag Laboratories Burlingame).

Flow cytometric analysis of bcl-2 protein or hsp70-bearing cells

Isolated leukemia cells or normal lymphocytes were fixed in $4 \%$ paraformaldehyde in phosphate-buffered saline (PBS, pH 7.4) for 20 minutes on ice and then washed once in PBS with $3 \%$ fetal bovine serum and $0.1 \%$ sodium azide (washing buffer). The fixed cells were permeabilized in $0.1 \%$ Triton X-100 in Tris-buffered saline (pH 7.4) for 5 minutes on ice. After three washes in a washing buffer, these fixed, permeabilized cells were incubated with bcl-2 protein-hsp70, or irrelevant control antibody for 20 minutes on ice, washed twice in a washing buffer, and incubated for 5 minutes with goat IgG to block unbound sites of the second antibody. The bcl-2 protein was used at a final concentration of $3 \mu \mathrm{g} / \mathrm{ml}$, and hsp70 was added to the cells at a dilution of 1:500. After being washed once in washing buffer at a 1: 100 dilution, FITC-labeled goat anti-mouse IgG was added to the cells for 20 minutes on ice. After the cells were washed twice, PI was added. Two-color 
immunofluoresence analyses were performed with a flow cytometer (Becton Dickinson, san Jose, California).

Flow cytometric analysis of CD19 and annexin $V$

Isolated leukemia cells or normal lymphocytes were resuspended at $1 \times 10^{6} / \mathrm{ml}$ in RPMI 1640 containing $10 \%$ fetal calf serum and cultured in a $5 \% \mathrm{CO}_{2}, 95 \% \mathrm{O}_{2}$, humidified atmosphere at $37^{\circ} \mathrm{C}$. After 24 hours, $15 \mu 1$ of CD19 antibody was added to the cells for 15 minutes on ice and after two times washing, the cells were resuspended in binding buffer (10mM HEPES/ NaOH pH 7.4; $140 \mathrm{mM} \mathrm{Nacl;} 2.5 \mathrm{mM} \mathrm{CaCl} \mathrm{Cl}_{2}$ and incubated with FITc-labeled human recombinant Annexin $V$ at a final concentration of $2 \mu \mathrm{g} / \mathrm{ml}$ Annexin V. The mixture was kept on ice in the dark for 5 minutes. Then two-color immunofluorecence analyses were performed using a FACScan flow cytometer.

Statistical analysis

Comparison of bcl-2 protein, hsp70 expression in normal lymphocytes and B-CLL cells was performed using the unpaired Student's t-test.

\section{Results}

\section{Expression of hsp70 and bcl-2 protein}

The flow cytometric data are shown in Table 1. Expression of hsp 70 in B-CLL cells increased more significantly $(p=0.030)$ than in normal lymphocytes and ALL cells. Expression of bcl-2 protein also increased more $(p=0.0504)$ in B-CLL cells compared with normal and ALL cells (Fig. 1). The normal lymphocyte staining hsp70 fluorescence showed a single narrow peak, while in the leukemic cells from ALL and B-CLL patients the pattern of hsp70 staining was relatively broad (Fig. 2a). The cell staining bcl-2 protein fluorescence showed a single narrow peak and indicated a homogenous pattern of bcl-2 protein expression in normal and leukemic cells (Fig. 2b). These results indicate that the fluorescence pattern of bcl-2 protein is not associated with the difference between normal and leukemic cells or mature and immature cells. In contrast, the fluorescence pattern of hsp70 seems to be related to the difference of normal and leukemic cells.

The relation between cell cycle and expression of hsp70 and bcl-2 protein

We analyzed the cell cycle from a DNA histoqram. Apoptotic cells have a lower DNA content than normal cells aod their presence is distinguished by the appearance of a subdiploid peak of lower fluorescence intensity. In our study, the normal lymphocytes, B-CLL cells and ALL cells stained as a single narrow peak of normal G0/G1. A subdiploid peak did not appear in normal lymphocytes, B-CLL cells and ALL cells at day 0 (Fig. 3). This result indicates that the cell cycle is not associated in either normal lymphocyte or leukemic cells.

Sensitivity of normal and leukemia cells to apoptosis in vitro

Using annexin $\mathrm{V}$ as a specific marker of apoptosis, we studied apoptotic cells from day 0 to day 1 in the absence of exogenous growth factors. Annexin $\mathrm{V}$ positive cells mean apoptotic cells. The mean percentage of apoptotic leukemia cells in B-CLL was $2.43 \%$ at day 0 and $16.93 \%$ at day 1 , and that in normal B lymphocytes was $0.46 \%$ at day 0 and $3.32 \%$ at day 1 (Table 2 ). These findings show that sensitivity of B-CLL cells to apoptosis in vitro is higher than that of normal B lymphocytes. Although this study is limited in number, the data in ALL cells was $7.63 \%$ at day 0 and $6.94 \%$ at day 1. The data of two-color FACScan is shown in Fig. 4. 
Table 1 Bcl-2 and hsp70

(a) Healthy volunteers

\begin{tabular}{ccc}
\hline Patient & bcl-2 $($ S.I $)$ & HSP70 $($ S.I $)$ \\
\hline & 4.93 & 6.07 \\
& 2.91 & 19.87 \\
& 3.17 & 11.54 \\
& 4.49 & 10.77 \\
& 2.97 & 17.3 \\
& 3.22 & 14.96 \\
& 2.86 & 23.13 \\
& 3.17 & 4.78 \\
& 2.75 & 20.59 \\
& 5.17 & 12.57 \\
\hline Mean signal & 3.56 & 14.16 \\
\hline intensity & & \\
\hline
\end{tabular}

(b) B-CLL patients

\begin{tabular}{ccc}
\hline Patient & bcl-2 $($ S.I $)$ & hsp70 (S.I) \\
\hline & 7.52 & 82.33 \\
& 5.33 & 9.21 \\
& 8.75 & 47.88 \\
& 14.97 & 24.34 \\
& 4.05 & 4.99 \\
& 3.31 & 39.98 \\
& 2.76 & 3.02 \\
& 5.76 & 21.06 \\
& 2.7 & 27.93 \\
& 17 & 46.81 \\
& 28.96 & 17.44 \\
Mean signal & 2.73 & 44.1 \\
\hline intensity & 8.65 & 30.76 \\
\hline
\end{tabular}

(c) ALL patients

\begin{tabular}{cccc}
\hline Patient & FAB & bcl-2 $($ S.I $)$ & hsp70 $($ S.I $)$ \\
\hline & L2 & 4.72 & 3.63 \\
& L2 & 2.08 & 26.03 \\
L2 & 5.34 & 3.16 \\
& L1 & 3.1 & 4.93 \\
& L2 & 3.91 & 19.75 \\
& L2 & 6.36 & 14.37 \\
& L1 & 6.39 & 15.61 \\
& L2 & 2.28 & 17.62 \\
& L1 & 9.41 & 46.81 \\
& & 3.83 & 20.99 \\
& L2 & 3.5 & 16.81 \\
& & 2.36 & 7.04 \\
& & 2.86 & 4.22 \\
& & 3.36 & 13.54 \\
& L1 & 6.19 & 17.27 \\
& & 3 & 76.67 \\
& L1 & 3.02 & 3.52 \\
\hline Mean signal & & 4.22 & 14.23 \\
\hline
\end{tabular}

\section{Discussion}

The present study showed that both expressions of intracellular hsp70 and bc1-2 protein increased in the leukemia cells of B-CLL patients compared with healthy volunteers and ALL patients. 
$\mathbf{a}$

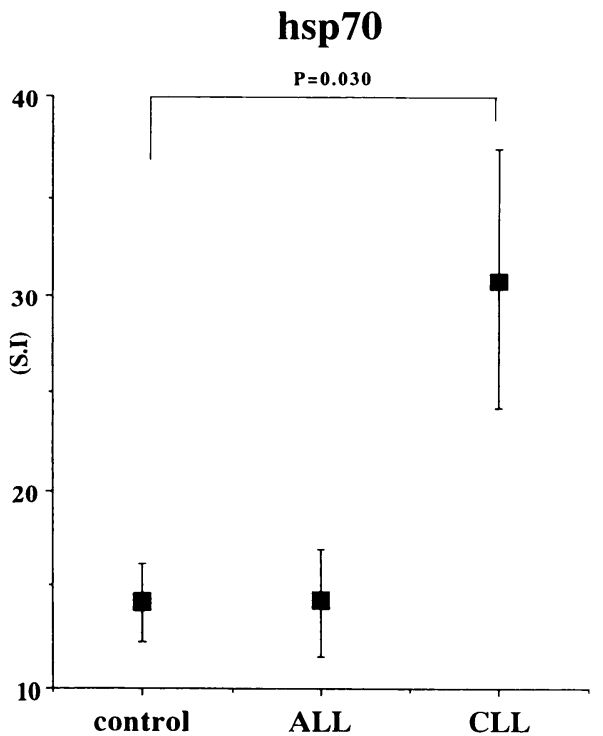

bcl-2

b

Fig. 1. (a) Intracellular hsp70 expression in B-CLL and ALL cells. Intracellular hsp70 expression increased significantly $(\mathrm{p}=0.030)$ in B-CLL cells.

(b) Inttacellular bc1-2 protein expression in B-CLL and ALL cells. Intracellular bc1-2 protein expression increased in B-CLL cells, although not significantly $(\mathrm{p}=$ 0.0504 ).

$\mathbf{a}$

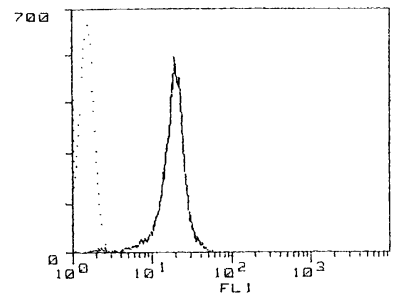

control

b

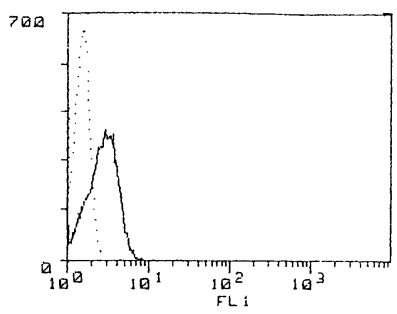

control

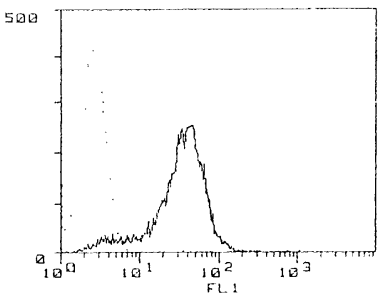

B-CLL

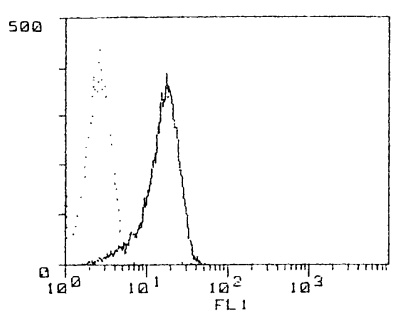

B-CLL

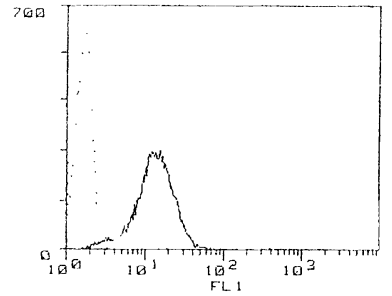

ALL

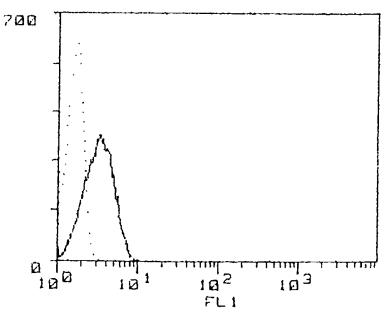

ALL

Fig. 2. Representative profile of (a) intracellular hsp70 (b) intracellular bc1-2 protein staining in leukemic cells from B-CLL and ALL patients. B-CLL cells express higher levels of intracellular hsp70 and bc1-2 protein. Negative control is indicated by dotted line. Heterogeneous expression of intracellular hsp70 is indicated by a broad staining peak compared with that seen for normal lymphocyte. Intracellular bc1-2 protein staining shows a homogenous pattern. 


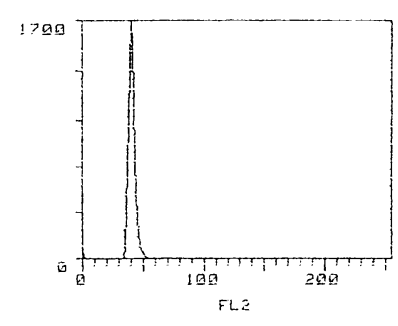

control

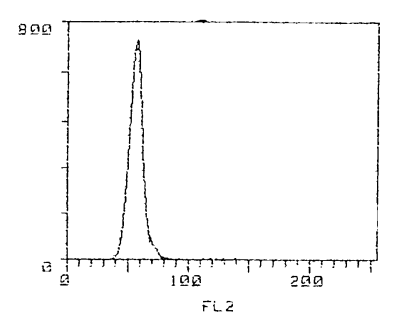

B-CLL

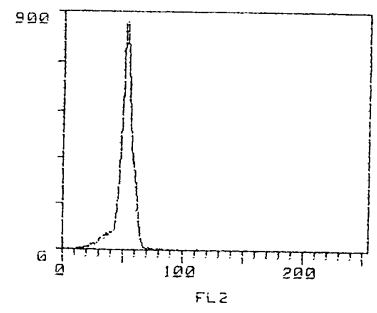

ALL

Fig. 3. Single narrow normal G0/G1 peak is seen in normal lymphocyte, B-CLL and ALL cells staining with propidium iodide by flow cytometry. Subdiploid apoptotic peaks are not seen in normal lymphocyte, B-CLL and ALL cells at day 0.

Hsp70 genes encode abundant heat-inducible 70kd hsp70s. The hsp70 genes are found in most cellular compartments of eukaryotes including nuclei, mitochondria, chloroplasts, endoplasmic reticulum and cytosol ${ }^{1}$. Hsp70 has an important role as a functional protein folding assembly, disassembly and degradation. Hsp70 is mainly known as molecular chaperones $^{2)}$. A number of studies have reported that hsp70 a lso plays a role in apoptosis as well as bc1-2. Several studies have demonstrated hsp70 overexptession in some tumors ${ }^{10,11)}$. Chant et al. reported that hsp70 expression increased in myeloid leukemia cells and the susceptibility of AML cells to in vitro apoptosis correlated with hsp70 overexpression $^{6,12)}$.

The bc1-2 gene was initially discovered because of its involvement in the $\mathrm{t}(14 ; 18)$ chromosomal translocation found in the majority of low grade follicular lymphoma ${ }^{13)}$. This gene encodes-a $26 \mathrm{kd}$ integral membrane protein that appears in mitochondria, nuclear and endoplasmic reticulum and blocks programmed cell death (apoptosis) ${ }^{14,15)}$. Bc1-2 is a protein that serves as key regulator of apoptosis, and it has been suggested that bc1-2 is a multifunctional protein. In simplistic terms, the bc1-2 protein is known to have two major types of functions. One is as an adaptor or docking protein that interacts with a variety of other proteins and helps to target them to defined places in the cell where bc1-2 resides. The other function is as a protein involved in pore or channel formation in membranesis ${ }^{16}$. Increased expression of bc1-2 is believed to exert a protective effect against apoptosis. Thus, increased expression of bc1-2 in B-CLL means low sensitivity of B-CLL cells to apoptosis.

Interestingly, our study demonstrates that in spite of high levels of bcl-2 protein expression of the in vitro culture, B-CLL cells undergo apoptosis easily. This discrepancy can be explained by the pore formation theory. An interesting aspect of this channel formation is that it probably requires at least dimerization if not higher order oligmerization of bc1-2 in membranes. Depending on the number of bc1-2 molecules that assemble in the membrane, the lumena of these channels may only be large enough to transport ions. Alternatively, these channels may be sufficiently large for transporting proteins. The three dimensional structure of bc1-2 reveals a striking similarity to the pore-forming domains of certain bacterial toxins; among these is diphtheria toxin, which is known for its ability to transport a protein across membranes. The explanation means that bc1-2 should form channels that are cytotoxic. Overexpression of bc1-2 at very high levels paradoxically can have a cytotoxic rather than cytoprotective function. As mentioned above, hsp70 is a 
a

CD19

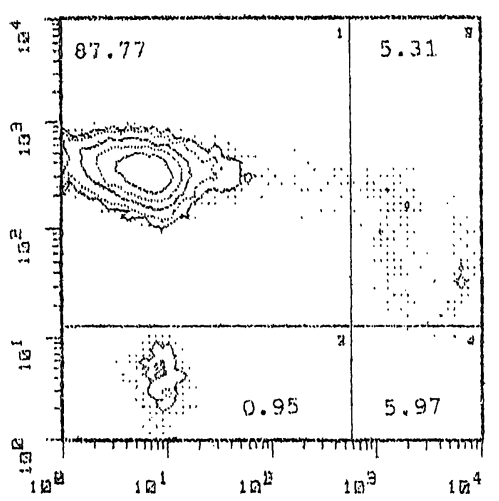

ANNEXIN $V$

b

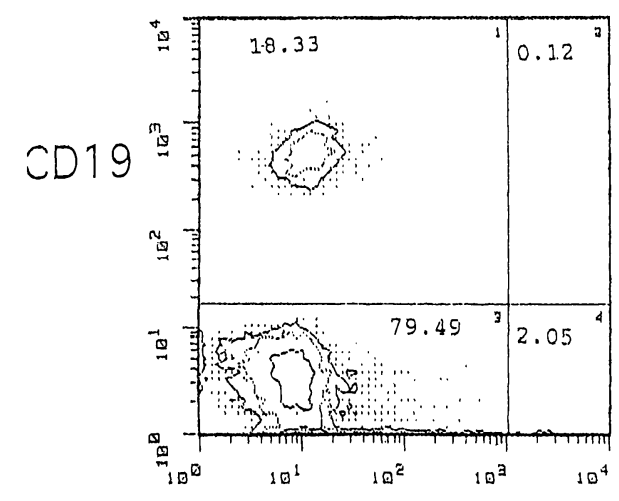

ANNEXIN $V$
DAY 1

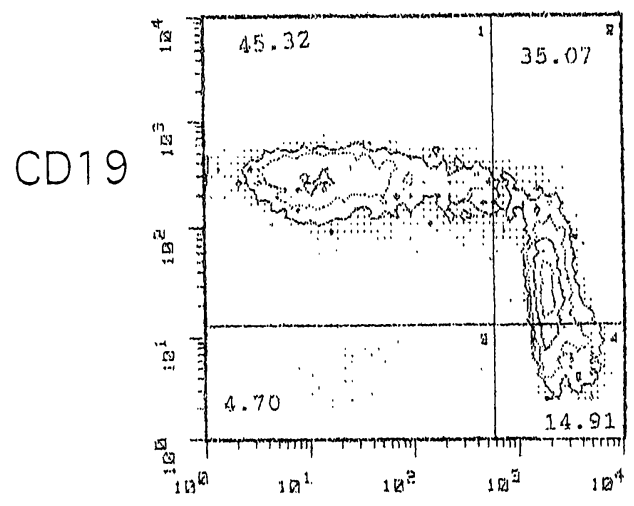

ANNEXIN $\vee$

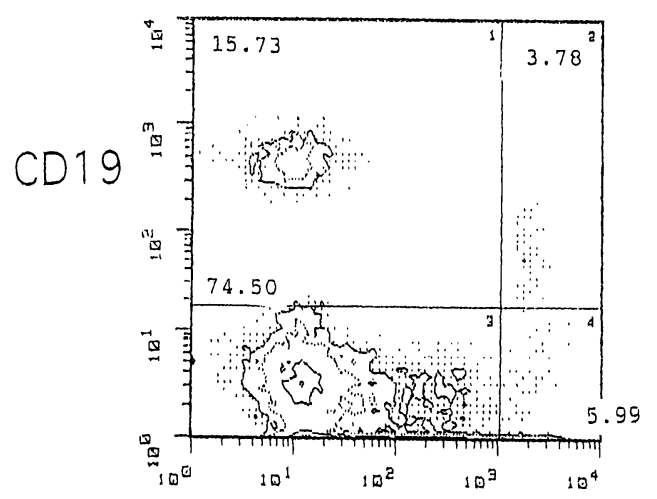

ANNEXIN $\vee$

Fig. 4. Apoptotic cells in (a) B-CLL patients (b) healthy volunteers at day 0 and day 1. CD19 positive cells are normal B lymphocyte and B-CLL cells. Annexin V, specific antibody for apoptosis, positive cells mean apoptotic cells. After 24 hours, the percentage of apoptotic cells in B-CLL was $48.71 \%$. In contrast, that in normal B lymphocyte was $3.78 \%$. Apoptotic cells increased more in B-CLL than in normal B lymphocyte.

multifunctional protein like bc1-2. We may consider the same theory, although, at present, we do not have a clear explanation. The challenge now is to sort out the relation between hsp70 and p53. Interestingly, hsp70 interacts with the mutant p53 ${ }^{21,22)}$. Hsp70 regulates folding from mutant p53 to wild type p53 conformation ${ }^{23)}$. In tumor cells, a hsp70-mutant p53 complex is found and blocks this conformation.

In conclusion, our study indicates that there is an inverse relationship between the high expression of hsp70 and bc1-2 protein and high sensitivity of B-CLL cells to apoptosis. Understanding more about the apoptosis mechanism in B-CLL ultimately will contribute to important of strategies for the treatment of B-CLL and other malignancies. 
Table 2 Apoptotic cell in 24-hour culture

\begin{tabular}{lcc} 
(a) healthy volunteers & \multicolumn{2}{c}{ apoptotic cell (\%) } \\
\hline & oh & $24 \mathrm{~h}$ \\
\hline & 1.11 & 2.97 \\
& 0.75 & 2.39 \\
& 0.12 & 3.78 \\
& 0.2 & 3.69 \\
& 0.1 & 3.77 \\
\hline mean & 0.46 & 3.32 \\
\hline
\end{tabular}

\begin{tabular}{lcc} 
(b) B-CLL patients & \multicolumn{2}{c}{ apoptotic cell $(\%)$} \\
\hline & oh & $24 \mathrm{~h}$ \\
\hline & 2.03 & 12.60 \\
& 3.99 & 5.44 \\
& 3.2 & 48.71 \\
& 2.48 & 9.20 \\
\hline mean & 2.43 & 16.93 \\
\hline \multirow{2}{*}{ (c) ALL patients } & apoptotic cell $(\%)$ \\
\hline & oh & $24 \mathrm{~h}$ \\
\hline & 9.66 & 7.82 \\
& 5.60 & 6.06 \\
\hline mean & 7.63 & 6.94 \\
\hline
\end{tabular}

\section{References}

1) Lindguist S and Craig EA : The heat shock proteins. Annu Rev Genet, 22 : 631-677 (1988)

2) Ellis RJ and Van der Vies SM: Molecular chaperones. Annu Rev Biochem. 60 : 321-347 (1991)

3) Yu-guan W, Xia Z, Kariya Y, Teshigawara $\mathbf{K}$ and Uchida $\mathbf{A}$ : inhibition of proliferation and induction of apoptosis by abrogation of heat-shock protein (hsp) 70 expression in tomor cells. Cancer Immunol Immunother, $40: 73-78(1995)$

4) Carper SW, Duffy JJ and Gerner EW : Heat snock proteins in thermotolerance and other cellular processes. Cancer Res, 47 : 5249-5255 (1987)

5) Ferrarini M, Helta S, Zocchi MR and Rugarli C: Unusual expression and localization of heat shock proteins in human tumor cells. Int $J$ Cancer, 51 : 613-619 (1992)

6) Yu-guan W, Xia Z, Kariya Y, Fukuta H, Teshigawara K and Uchida A : Induction of apoptosis by quercerin, involvement of heat shock protein. Cancer Res, $54: 4952-4957$ (1994)

7) Chant ID, Rose PE and Morris AG : Susceptibility of AML cells in vitro apoptosis cortelates with heat shock protein 70 (hsp70) expression. Br J Haematol, 93 : 898-902 (1996)

8) Binnet JL : Chronic lymphocytic leukemia : Recommendations for diagnosis, staging and response criteria. Ann Intern Med, 110 : 236-238 (1989)

9) Bennett JM : Proposals for the classification of the acute leukemias. Br J Haematol, 33 : 451-458 (1976)

10) Welch WJ and Suhan JP: Cellular and biochemical events in mammalian cells during after recovery from physiological stress. J cell Biol, 103 : 2035-2052 (1986)

11) Yufu $Y$, Nishimura J, Ideguchi $\mathbf{H}$ and Nawata $\mathbf{H}$ : Heterogeneity of the synthesis of heat shock proteins in human leukaemic cells. Br J Cancer, 62 : 65-68 (1990)

12) Chant ID, Rose PH and Morris AG: Analysis of heat-shock protein expression in myeloid leaukaemia cells by flow cytometry. Br J Haematol, $90: 163-168$ (1995) 
13) Tujimoto $\mathrm{Y}$, Cossman $\mathrm{J}$ and Jaffe $\mathrm{E}$ : Involvement of the bc1-2 gene in human follicular lymphoma. Science, 228 : 1440-1443 (1985)

14) Hockenney D, Nunez G, Milliman C, Schreider RD and Korsmeyer SJ : Bc1-2 is an inner mitochondrial membrane protein that blocks programmed cell death. Nature, $348: 334-336$ (1990)

15) Vaux DL, Cory S and Adams JM: Bc1-2 gene promotes haematopoietic cell survival and cooperates with c-myc to immortalize pre-B cells. Nature, 335 : 440-442 (1988)

16) Kipps TJ : Immunoglobulins and B cell fate. Hematol Cell Ther, 39 : S21-S22 (1997)

17) Hainaut $P$ and Milner $J$ : Interaction of heat-shock protein with $p 53$ translated in vitro: evidence for interaction with dimeric p53 and for a role in the regulation of p53 conformation. EMBO J, 11: 3513-3520 (1992)

18) Pinhasi-Kimhi O, Michalovitz D, Ben-Zeev A and Oren $\mathbf{M}$ : Specific interaction between the p53 cellular tumor antigen and major heat shock proteins. Nature, 320 (1986)

19) Ullrich SJ, Mercer WE and Appella E: Human wild type p53 adopts a unigue conformational and phosphorylation state in vivo during growth arrest of glioblastoma cells. Oncogene, $7: 1635-1643$ (1992)

[Received January 16, 1998 : Accepted February 5, 1998] 